\section{Susceptibility to Various Antimicrobial Agents and Tolerance to Methicillin of Staphylococcus aureus Isolates from Cystic Fibrosis Patients}

Pseudomonas aeruginosa, Staphylococcus aureus and Haemophilus influenzae are the pathogenic bacterial species most frequently detected in the respiratory tract of patients with cystic fibrosis (CF). To minimize progression of pulmonary destruction, acute exacerbations in CF demand intensive antimicrobial chemotherapy (1). Since persistent colonization and infection with identical types of Staphylococcus aureus have been described (2), frequent and prolonged treatment with various antimicrobial agents may influence resistance and tolerance in strains. Tolerance to penicillin has been proposed as one possible explanation for the poor response of some streptococcal infections to penicillin therapy (2). In this study we tested the activity of various antimicrobial agents against Staphylococcus aureus isolates from patients with CF. In addition tolerance to methicillin in the isolates was investigated.

Fifty-two isolates of Staphylococcus aureus were recovered from sputum of 52 patients with $C F$. The strains were identified biochemically by means of the API-STAPH identification system and stored at $-70^{\circ} \mathrm{C}$ until tested. Additional tests included a slide coagulase test, a catalase test and mannitol fermentation.

Detection of beta-lactamases was performed as follows. Nitrocefin $(0.1 \mathrm{ml})$ was placed in a microdilution well and a bacterial suspension yielding $10^{7} \mathrm{cfu} / \mathrm{ml}$ was added. Production of beta-lactamases was established if the solution turned a deep red within ten minutes.

Antimicrobial agents were obtained as a standard laboratory powder from the manufacturers and stored and handled as recommended. MICs were determined by an agar dilution technique. Freshly prepared serial twofold dilutions of antibiotic were incorporated in Diagnostic Sensitivity Test agar (Oxoid, UK). Agar supplemented with 4\% $\mathrm{NaCl}$ was used to define methicillin susceptibility (3). Final concentrations ranged from 0.006 to $25.6 \mathrm{mg} / \mathrm{l}$ (for penicillin up to $410 \mathrm{mg} / \mathrm{l}$ ). Plates were inoculated with a multipoint inoculator, which delivered a final inoculum of $10^{4} \mathrm{cfu}$ per spot ( $1 \mu$ l of liquid) from overnight Mueller-Hinton broth (Oxoid) cultures adjusted to $10^{8} \mathrm{cfu} / \mathrm{ml}$ using a barium sulphate standard and diluted $10^{-1}$. Inoculated plates were incubated at $35^{\circ} \mathrm{C}$ for 24 hours. The MIC was defined as the lowest con- centration of antimicrobial agent which inhibited visible growth. Staphylococcus aureus strains ATCC 25923 and ATCC 29213 were included as control organisms in all sets of inoculations, and the MICs were consistent with established values within one twofold dilution.

Tolerance to methicillin was determined using the gradient technique of Kim and Anthony (4). Briefly, $40 \mathrm{ml}$ of Mueller-Hinton agar (Difco) supplemented with $20 \mathrm{mg} / \mathrm{l}$ methicillin was allowed to gel in a $15 \times 15 \times 1.5 \mathrm{~cm}$ square petri dish which was placed at an angle to form a wedge. Once cooled, $60 \mathrm{ml}$ of the same agar, but without antibiotics, was overlaid, and allowed to set on a level surface resulting in graduated concentrations nominally ranging from 0 to $20 \mathrm{mg} / \mathrm{l}$. Late-logarithmic cultures were prepared and applied undiluted using cotton wool swabs. Up to six strains together with a non-tolerant and a tolerant control were inoculated as parallel streaks beginning at the lowest concentration along the axis of the gradient. As expected, there was no apparent growth after 24 hours and an impression of the surface was made using a sterile velvet pad which was applied to a methicillin-free plate. This was incubated for 24 hours and the result growth was compared with that of the controls. Tolerance was defined as any growth which occurred beyond that of the nontolerant control along the line of inoculation.

The results are summarized in Table 1 and Table 2. Fourty-nine isolates produced beta-lactamases. The penicillin and methicillin MIC modes for these strains were 12.8 and $1.6 \mathrm{mg} / \mathrm{l}$ respectively. Methicillin resistant strains of Staphylococcus aureus were not found. On a weight basis imipenem and rifampicin were the most active of the agents tested, inhibiting $100 \%$ of the isolates at $0.025 \mathrm{mg} / \mathrm{l}$ and $0.05 \mathrm{mg} / \mathrm{l}$ respectively. At clinically attainable serum concentrations flucloxacillin, cefamandole and cefuroxime had similar activity. With the exception of erythromycin, clindamycin and fusidic acid, MIC ranges were within one (imipenem, vancomycin) to four (ciprofloxacin, gentamicin) twofold dilutions. However, it should be noted that the wider MIC ranges observed for erythromycin, clindamycin and fusidic acid were due to a single isolate. The gentamicin MIC for the 52 isolates was $0.4 \mathrm{mg} / \mathrm{l}$ or less. In general, MICs of the drugs tested were within the therapeutic range. Six strains $(11.2 \%)$ appeared to be tolerant to methicillin. The proportion of tolerant strains identified in this study, i.e. $11.2 \%$, is similar to that of $12.5 \%$ reported by Coessens et al. (5) in non-CF populations of Staphylococcus aureus. We therefore conclude that frequent therapy in 
Table 1: In vitro activity of penicillin and methicillin against 52 isolates of Staphylococcus aureus obtained from 52 patients with cystic fibrosis.

\begin{tabular}{lccccc}
\hline $\begin{array}{l}\text { Production of } \\
\text { beta-lactamases }\end{array}$ & \multicolumn{2}{c}{ Penicillin MIC $(\mathrm{mg} / \mathrm{l})$} & & \multicolumn{2}{c}{ Methicillin MIC (mg/l) } \\
\cline { 2 - 3 } \cline { 5 - 6 } & Range & Mode & & Range & Mode \\
\hline Negative $(\mathrm{n}=3)$ & 0.05 & 0.05 & & $1.6-3.2$ & 1.6 \\
Positive $(\mathrm{n}=49)$ & $0.8-102.4$ & 12.8 & & $0.8-12.8$ & 1.6 \\
\hline
\end{tabular}

Table 2: In vitro activity of various antimicrobial agents against 52 isolates of Staphylococcus aureus obtained from 52 patients with cystic fibrosis.

\begin{tabular}{|c|c|c|c|c|c|c|c|c|c|c|c|}
\hline \multirow{2}{*}{$\begin{array}{l}\text { Antimicrobial } \\
\text { agent }\end{array}$} & \multicolumn{11}{|c|}{ Cumulative pereentage of isolates inhibited at given concentration ( $\mathrm{mg} / \mathrm{l})$} \\
\hline & $\leq 0.006$ & 0.012 & 0.025 & 0.05 & 0.1 & 0.2 & 0.4 & 0.8 & 1.6 & 3.2 & 26.4 \\
\hline Flucloxacillin & & & & & 13 & 73 & 100 & & & & \\
\hline Imipenem & & 60 & 100 & & & & & & & & \\
\hline Cefamandole & & & & & & 23 & 94 & 100 & & & \\
\hline Cefuroxime & & & & & 6 & 27 & 100 & & & & \\
\hline Gentamicin & & & 2 & 2 & 10 & 96 & 100 & & & & \\
\hline Erythromycin & & & & 2 & 10 & 96 & 96 & 96 & 98 & 98 & 100 \\
\hline Clindamycin & & & 2 & 12 & 96 & 98 & 98 & 98 & 98 & 98 & 100 \\
\hline Rifampicin & & 8 & 87 & 100 & & & & & & & \\
\hline Fusidic acid & & & & 46 & 98 & 98 & 98 & 98 & 98 & 98 & 100 \\
\hline Vancomycin & & & & & & & 10 & 100 & & & \\
\hline Teicoplanin & & & & & & 4 & 13 & 100 & & & \\
\hline Ciprofloxacin & & & & & 2 & 4 & 87 & 100 & & & \\
\hline Flcroxacin & & & & & & 6 & 98 & 100 & & & \\
\hline
\end{tabular}

CF does not result in higher tolerance. Whether tolerance plays a role or not in the clinical outcome in CF has never been determined. Using the simple and reliable method described here it should now be possible to determine this.

\section{A.M. Horrevorts $1 *$ J.W. Mouton ${ }^{2}$}

${ }^{1}$ Department of Medical Microbiology, University Hospital Nijmegen, Geert Grooteplein Zuid 24, 6524 GA, Nijmegen, The Netherlands.

${ }^{2}$ Department of Clinical Microbiology and Antimicrobial Therapy, University Hospital Rotterdam, The Netherlands.
2. Goering RV, Bauernfeind A, Lenz W, Przyklenk B: Staphylococcus aureus in patients with cystic fibrosis: an epidemiological analysis using a combination of traditional and molecular methods. Infection 1990, 18: $57-60$.

3. Jorgensen JH: Laboratory and epidemiologic experience with methicillin-resistant Staphylococcus aureus in the USA. European Journal of Clinical Microbiology 1986, 5: 693-696.

4. Kim KS, Anthony BF: Use of penicillin-gradient and replicate plates for the demonstration of tolerance to penicillin in streptococci. Journal of Infectious Diseases 1983, 148: 488-491.

5. Goessens WHF, Fontijne P, van Raffe M, Michel MF: Tolerance percentage as a criterion for the detection of tolcrant Staphylococcus aureus strains. Antimicrobial Agents and Chemotherapy 1984, 25: 575578.

\section{References}

1. Mouton JW, Kerrebijn KF: Antibacterial therapy in cystic fibrosis. Medical Clinics of North America 1990, 74: $837-850$. 